\title{
Neuroprótesis: Hacia un futuro de cuerpos cibernéticos
}

\author{
Walter Castellanos Pantoja \\ Ingeniería Industrial n. 26, 2008, ISSN 1025-9929, pp. 251-266
}

\begin{abstract}
Resumen: Hace veinte años, Masamune Shirow pronosticó que en un futuro cercano será posible crear entidades cibernéticas mediante la integración de dispositivos biomiméticos al cuerpo humano. Muchas veces la realidad supera la ficción, pero ¿pueden realmente los avances tecnológicos confirmar las visiones de Shirow? En el presente artículo se presenta el estado del arte de las neuroprótesis, las mismas que permiten la mejora de la calidad de vida de pacientes usando dispositivos artificiales, ya sea para restaurar algunos sentidos perdidos como el oído, la visión e, incluso, las funciones cognitivas.
\end{abstract}

Palabras clave: Coclear, implante, implante cerebral, neuroprótesis, organismo cibernético, prótesis cognitiva, visión artificial.

\section{Neuroprosthesis: Towards a future of cybernetic bodies}

Abstract: 20 years ago, Masamune Shirow predicted that in a close future, it will be possible to create cyborg entities, integrating biomimetic devices to the human body. Many times the reality exceeds the fiction, but can the technological advances really confirm the Shirow's visions? In the present article, the state of the art of neuroprosthesis is described, the same ones that improve the patient's life quality by using artificial devices which allow the recovery of lost senses like hearing, vision and even cognitive function.

Keywords: Artificial vision, brian implant, cochlear, cognitive prosthesis, cyborg, implant, neural prosthesis. 


\section{INTRODUCCIÓN}

El vertiginoso avance tecnológico y la miniaturización de componentes electrónicos en las últimas décadas nos hace pensar en la necesidad de una interacción cada vez más íntima entre el cuerpo humano y la tecnología. Es así que en los años setenta del siglo pasado ya se vislumbraba la posibilidad de reemplazar partes del cuerpo del astronauta americano Steven Austin, luego de que este sufriera un accidente, por prótesis biónicas, en la serie de televisión The six million dollar man. También en 1989, el escritor japonés Masamune Shirow, en su obra Ghost in the Shell, llevada al cine con el mismo título (1996), preveía un futuro en el que los implantes biónicos para el perfeccionamiento del cuerpo serán cada vez mayores, de manera tal que en algunos casos solo el pensamiento humano estará presente en cuerpos cibernéticos.

¿Son acaso estas propuestas realidad o puramente ficción? De acuerdo con algunas definiciones del término "organismo cibernético" (Cyborg), la conexión física y metafísica del ser humano con la tecnología ya ha empezado.

En efecto, en la actualidad podemos encontrar implantes que permiten monitorear órganos como el corazón, pueden enviar mensajes de texto a un celular o a un correo electrónico, almacenar información del estado del paciente para su futura evaluación, restaurar el oído o la visión y otros que procesando los impulsos neuronales y enviando señales eléctricas pueden mover prótesis biónicas.

\section{NEUROPRÓTESIS}

La neurociencia analiza la posibilidad de reemplazar algunas funciones del sistema nervioso usando dispositivos artificiales. Esto se logra mediante la creación de modelos matemáticos biológicamente realistas de las funciones del cerebro, la producción de microchips biomiméticos que incorporan estos modelos y su integración al sistema nervioso central.

El dispositivo más usado en neuroprótesis es el implante de cóclea, utilizado para restaurar el oído; sin embargo, también existen implantes que permiten restaurar la visión e incluso funciones cognitivas, emulando al cerebro en el último caso. 
La transmisión sináptica también ha sido analizada mediante la utilización de neuroprótesis con excelentes resultados, lográndose traducir los impulsos neuronales y enviando señales por radiofrecuencia se puede mover el mouse de una PC, una silla de ruedas eléctrica y hasta prótesis biónicas. Las conexiones sinápticas de los nervios 'redireccionados' hacia electrodos también permiten captar sensaciones de los miembros perdidos.

\section{EL IMPLANTE AUDITIVO}

El implante auditivo o implante coclear de oído trabaja usando la estructura tonotópica de la membrana basilar del oído interno. Esta estructura, también conocida como mapa de frecuencia/posición, permite que el oído filtre las frecuencias para que el cerebro pueda procesar la información acústica. En un oído normal, las vibraciones acústicas se sitúan en el área cercana a las vibraciones resonantes de la membrana basilar dentro del laberinto.

Los sonidos de alta frecuencia no atraviesan la membrana, pero sí los sonidos de frecuencia baja. Se pueden distinguir sonidos sobre una gama de diez octavas, desde 20 hasta $20 \mathrm{khz}$. Mientras que la relación entre el sonido más intenso y el sonido más débil es de 120 decibeles.

El movimiento de células ciliadas localizadas cerca de la membrana basilar genera impulsos eléctricos en la rama utricular del nervio vestibular. El cerebro interpreta la actividad del nervio y determina el área de la membrana basilar que está resonando, y por consiguiente la frecuencia audible que está escuchándose.

En la figura 1 se muestra el esquema de un implante de cóclea, en el cual el sonido es detectado por un micrófono y la señal es convertida en una señal digital. El procesador externo (1) envía una señal de radiofrecuencia al procesador (2) en el implante interno (3), donde la señal es convertida en impulsos eléctricos para activar unos electrodos (4) que estimulan el nervio auditivo y el cerebro percibe las señales como si fuera un oído normal. 
Figura 1

Esquema del implante de cóclea

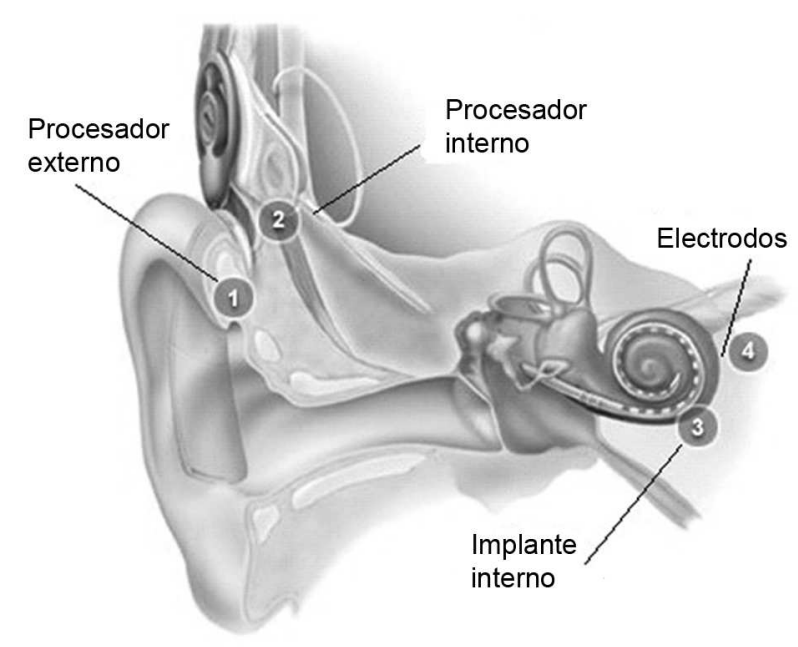

Fuente: University of Medicine and Dentistry of New Jersey.

Existen varios algoritmos para el procesamiento de la señal; así, en el modo más simple, la amplitud de la señal acústica se divide entre el número de electrodos y el voltaje resultante se aplica a cada uno de los electrodos. Los algoritmos de procesamiento, en la práctica, pueden ser más sofisticados, ya que la aplicación simultánea de voltaje a cada uno de los electrodos puede causar que fluyan corrientes desbalanceadas entre los electrodos. Esto podría estimular los nervios de manera indeseable y dañar el tejido, así como los electrodos, electrocutando esencialmente las células nerviosas individuales.

$\mathrm{El}$ algoritmo de Fourier usa filtros pasabandas para dividir la señal en diferentes bandas de frecuencia. El procesador selecciona cierta cantidad de las salidas más fuertes de los filtros. El número exacto de salidas depende de varios factores, entre ellos el número de electrodos implantados, tal como se muestra en la figura 2. Este algoritmo provee énfasis a la transmisión de los aspectos temporales del mensaje. 


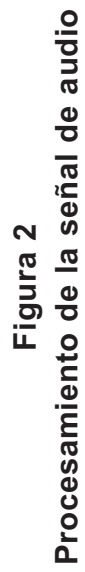

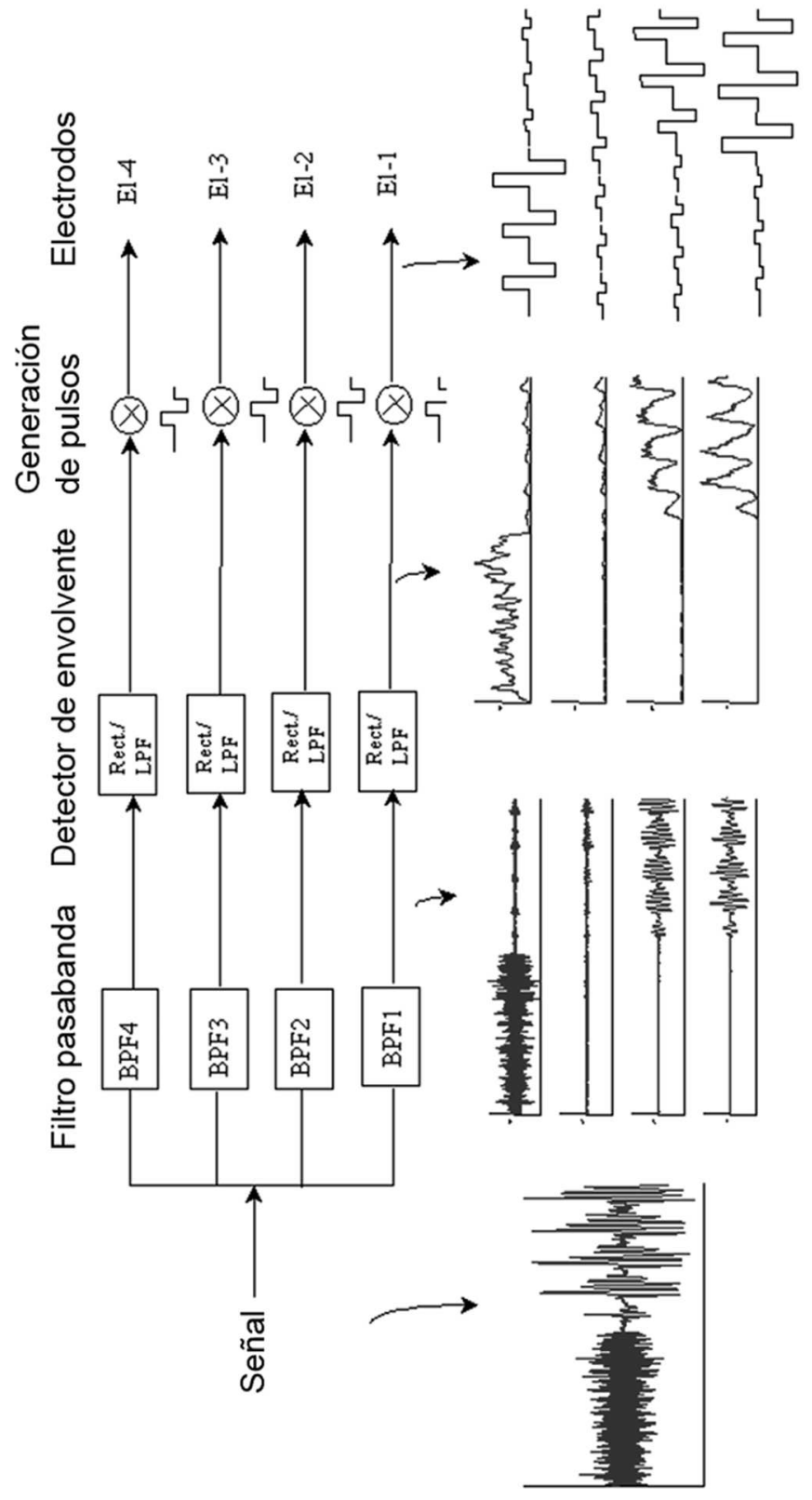

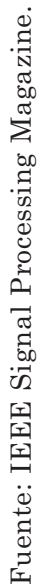


Otras estrategias de extracción de características usan rasgos que son comunes a todas las vocales. Cada vocal tiene una frecuencia fundamental (el pico de frecuencia más baja) y frecuencias secundarias (los picos con las frecuencias más altas). Los algoritmos mencionados intentan reconocer la vocal y dar énfasis a sus rasgos, y estas estrategias dan énfasis a la transmisión de aspectos espectrales del mensaje. Las estrategias de extracción de características no son muy usadas.

En la actualidad el microchip de procesamiento de señal está diseñado para manejar cierta cantidad de actualizaciones de software, que permiten usar las ventajas de nuevas técnicas de procesamiento sin intervención quirúrgica.

En el 2006, cerca de 100 mil personas en el mundo usaban este tipo de implante, según investigaciones de la Universidad de Michigan. En la figura 3 se muestra una típica prótesis de oído.

\section{Figura 3}

Prótesis típica de oído

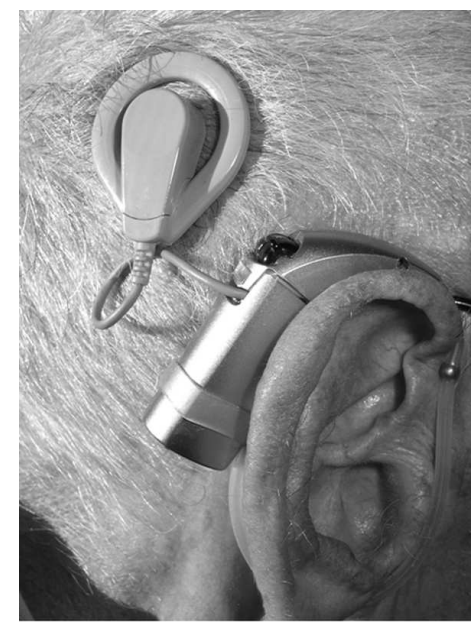

Fuente: Hearingloss. 
Figura 4

Esquema del implante de retina

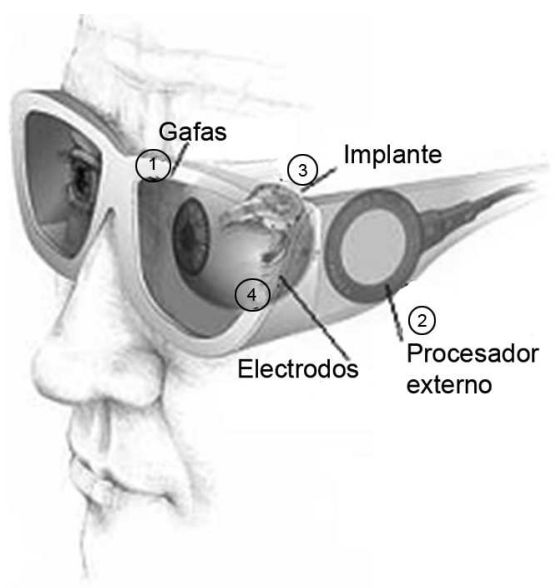

Fuente: MedGadget.

\section{EL IMPLANTE DE VISIÓN}

En un ojo normal, la información de luz es captada por los fotorreceptores situados en la retina. Esta información se transmite a las células ganglionares, donde se interpreta y se envía al cerebro a través del nervio óptico.

En un implante de retina los fotorreceptores que dejan de funcionar en la retina dañada son reemplazados por conos y bastones artificiales.

En la figura 4 se muestra el esquema de un implante de retina, en el que una microcámara de video montada sobre unas gafas (1) transmite la imagen a un procesador externo (2); la señal es procesada y transmitida por radiofrecuencia al procesador del implante interno (3) mediante el cual un arreglo de electrodos (4) en las células ganglionares envía impulsos al nervio óptico, que transmite la información al cerebro.

En febrero del 2007, la agencia federal de reglamentación de los productos farmacéuticos (FDA) de Estados Unidos aprobó el ensayo clínico de prótesis conectadas al sistema nervioso para restaurar la visión. El Instituto de Ojos de la Escuela de Medicina de la Universidad 
de California anunció el éxito de la primera prueba clínica sobre un pequeño grupo de seis pacientes que usaron una nueva generación de prótesis, Argus II Retinal Prosthesis System, dejando atrás la primera fase de los implantes, la que empezó en el 2002.

$\mathrm{El}$ arreglo que estimula el nervio óptico consta de 60 electrodos, los mismos que incrementan la resolución de la imagen en comparación a su versión anterior, que solo usaba 16 electrodos. Otros tipos de implantes usan un arreglo de microfotodiodos en vez de la cámara. El objetivo en este caso es inducir la regeneración de la retina mediante estimulación eléctrica.

Las estrategias de procesamiento avanzado de implante de retina propuesto por la Universidad de Bonn procesan la señal eléctrica proveniente de la cámara de video y la envían a un sistema virtual central, el cual -mediante un algoritmo inteligente o codificador de retina (Retinal Encoder) compatible con el sistema visual del cerebro y con un mecanismo de aprendizaje en proceso continuo- regula la señal de salida de la cámara de modo que cada paciente en particular puede percibir la imagen de una manera distinta, tal como se muestra en la figura 5.

En la figura 6 se muestra una prótesis de visión y el ensayo del codificador de retina. Al principio, el codificador no conoce el lenguaje del córtex visual. Posteriormente, el software convierte la imagen sin procesar en una serie de "dialectos" aleatorios, compatibles con el córtex visual y el paciente selecciona la forma más adecuada.

\section{EL IMPLANTE DEL MIEMBRO SUPERIOR}

El miembro superior está inervado por los ramos ventrales de los nervios cervicales quinto, sexto, séptimo y octavo y del primero torácico, que forman el plexo braquial. El quinto cervical suele recibir una contribución al plexo procedente del cuarto cervical y el segundo dorsal puede enviar fibras para unirse con el primero.

Los nervios supraclaviculares externos, procedentes del tercero y cuarto ventrales cervicales, inervan parte de la piel de la región del hombro. Las fibras eferentes del sistema simpático se distribuyen por los vasos sanguíneos, las glándulas sudoríparas y los músculos horripiladores o erectores pilosos. Las fibras preganglionares derivan del asta lateral de la médula espinal dorsal superior y forman sinapsis en 
Figura 5

Simulación de percepción durante la fase de aprendizaje

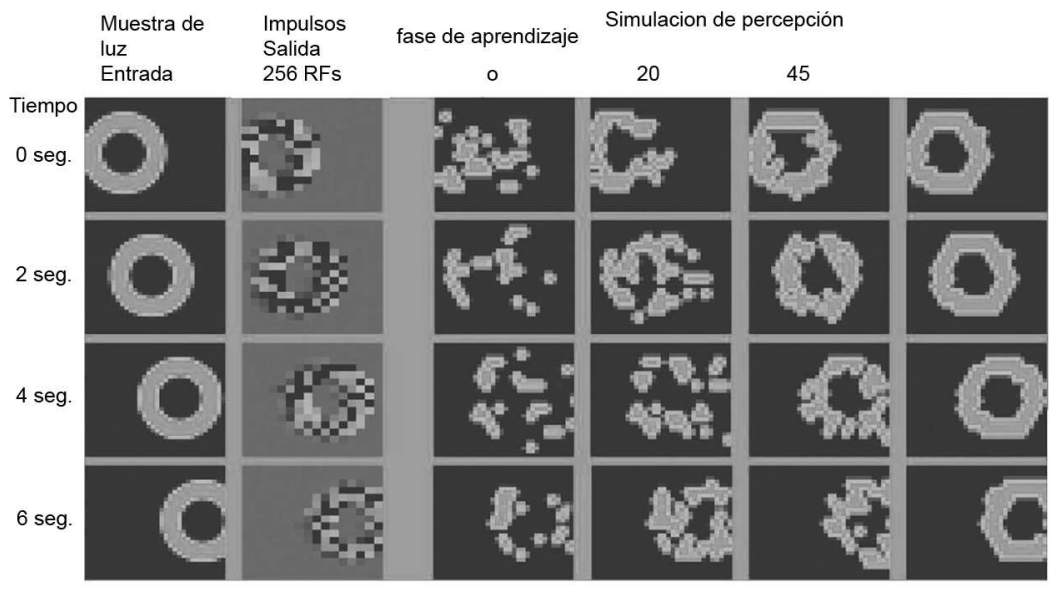

Fuente: Universidad de Bonn.

Figura 6

Ensayo de la prótesis de visión

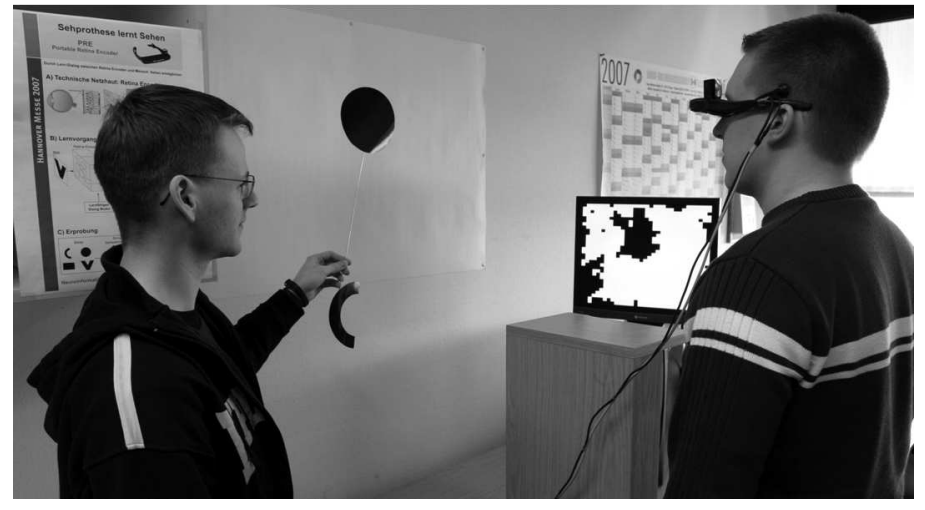

Fuente: Universidad de Bonn. 
los ganglios simpáticos cervical inferior, primario dorsal y cervical medio. Desde estos ganglios, las fibras posganglionares transmitidas principalmente por el tronco inferior del plexo braquial acompañan a los nervios periféricos y se distribuyen en sus campos de inervación.

En la figura 7 se muestra un esquema simplificado del flujo de impulsos cerebro/músculo, en el que se puede observar la trayectoria del estímulo del nervio sensitivo al cerebro y la trayectoria del estímulo del nervio motor al músculo.

Figura 7

Flujo de impulsos cerebro/músculo

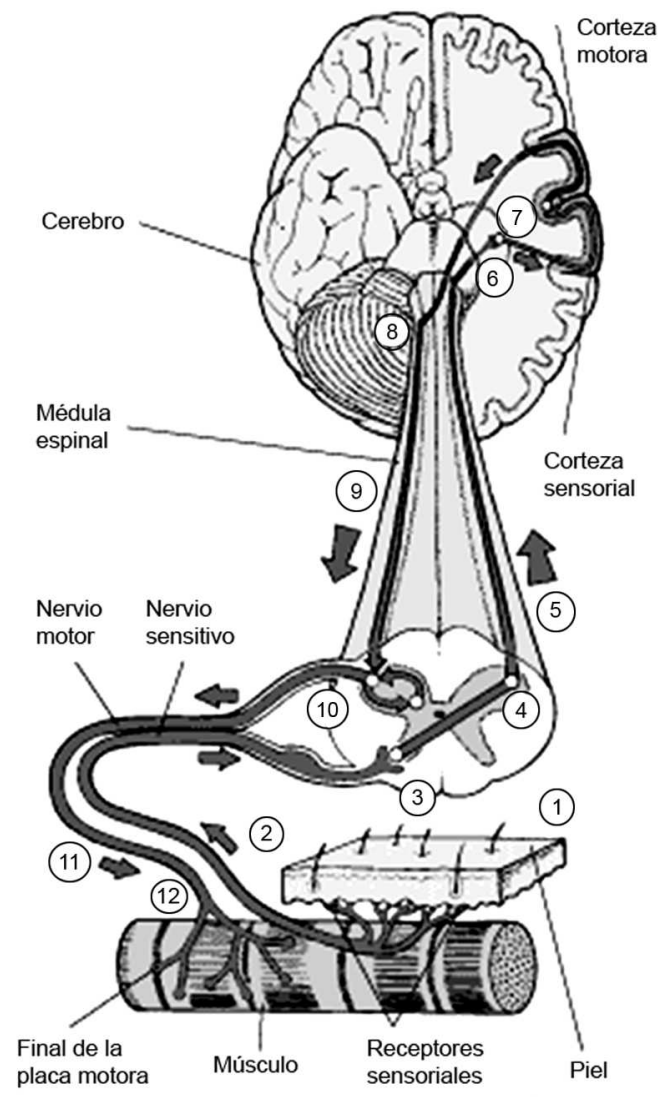

Fuente: Merck Sharp \& Dohme. 
Los receptores de los nervios sensitivos en la piel (1) detectan las sensaciones y envían una señal al cerebro. La señal (2) recorre el nervio sensitivo hasta la médula espinal. Una sinapsis (3) en la médula espinal conecta el nervio sensitivo a un nervio de la médula espinal. El nervio (4) cruza al lado opuesto de la médula espinal. La señal (5) asciende por esta y una sinapsis (6) en el tálamo conecta la médula espinal a las fibras nerviosas que llevan la señal (7) a la corteza sensorial. La corteza sensorial percibe la señal e impulsa a la corteza motora a generar una señal de movimiento.

El nervio (8) que lleva la señal cruza al otro lado en la base del cerebro, la señal (9) desciende por la médula espinal. Una sinapsis (10) conecta la médula espinal al nervio motor. La señal (11) a lo largo del nervio motor alcanza el final de la placa motora (12), desde donde estimula el movimiento muscular.

En agosto del 2006, Claudia Mitchell, de 26 años, se convirtió en la primera mujer a la que se le implantó un brazo biónico que le permite controlar partes del dispositivo exclusivamente por sus pensamientos. Claudia, quien perdió el brazo y parte del hombro tres años antes en un accidente, fue intervenida en el Instituto de Rehabilitación de Chicago por un grupo de ingenieros y médicos. Este brazo biónico pesa siete kilogramos y es una versión más sofisticada del brazo de Jesse Sullivan, a quien se le implantó el primer brazo biónico en el 2001.

En la figura 8 se muestran las conexiones del brazo biónico. Los movimientos de los músculos del pecho han sido reconectados a la sinapsis de los nervios (1) que una vez estuvieron conectados al miembro perdido. Esta técnica se conoce con el nombre de reinervación. Un arreglo de electrodos (3) situado en la correa capta las señales del cerebro.

El procesador (4) recibe los impulsos eléctricos y ejecuta movimientos del brazo como flexión del codo, apertura y cierre de la mano y giro de la muñeca, de modo que el movimiento del brazo se controla con el pensamiento. Este se mueve de la misma forma que lo hace un brazo normal y tiene seis motores para controlar igual número de movimientos, pero puede incrementarse hasta 22 .

Los nervios 'redireccionados' del pecho (5) también captan la sensación de tacto y ella siente como si le tocasen la mano. 
Los estudios para la determinación de los parámetros de la cinemática del brazo biónico consideran dos rotaciones en el hombro (flexión y abducción del húmero), una rotación en el codo (flexión del codo). El número de grados de libertad del brazo depende de la precisión que se desea obtener. La flexión y extensión del esterno-clavícula son despreciadas en algunos casos, debido a que estos no mejoran significativamente el espacio de trabajo. El brazo puede incluir también la mano completa y el movimiento de los dedos.

\section{IMPLANTE CEREBRAL}

La emulación de las funciones de procesamiento de los impulsos cerebrales del hipocampo neuronal para corregir el daño cerebral irreversible producido por accidentes o enfermedades como el Parkinson o el Alzheimer es posible mediante la utilización de la neuroprótesis. Algunas veces este implante es también llamado interfaz cerebro computador BCI (Brain Computer Interface). El término cerebro (brain) en este caso se usa para hacer referencia al sistema nervioso.

Este tipo de implante es cualitativamente diferente de los anteriores, ya que la zona neuronal dañada del hipocampo es reemplazada por un dispositivo artificial o neuronas de silicio. El hipocampo es una región del cerebro involucrada en la generación de memoria de largo plazo y es responsable del reconocimiento e identificación de características de objetos de una colección. La degeneración o daño del hipocampo produce desórdenes de la memoria.

El hipocampo funciona como un sistema de lazo cerrado, los estímulos del neocórtex entran a través del córtex entorrinal y se propagan hacia las subregiones intrínsecas del hipocampo para regresar nuevamente al neocórtex. La trayectoria intrínseca consiste en conexiones en cascada transversales al eje del hipocampo, de una manera similar a un juego de circuitos interconectados en paralelo.

En la figura 9 se muestra un esquema simplificado del implante cerebral. El implante consiste en un arreglo de sensores que recibe señales de la actividad neuronal del hipocampo y luego de procesar la información mediante algoritmos de extracción de características en un microchip biomimético, envía señales de salida a un arreglo de electrodos que estimula el cerebro. El microchip está diseñado sobre la base de un modelo matemático biológicamente realista de las funciones del cerebro. 
Figura 8

Conexiones del hrazo hoonico

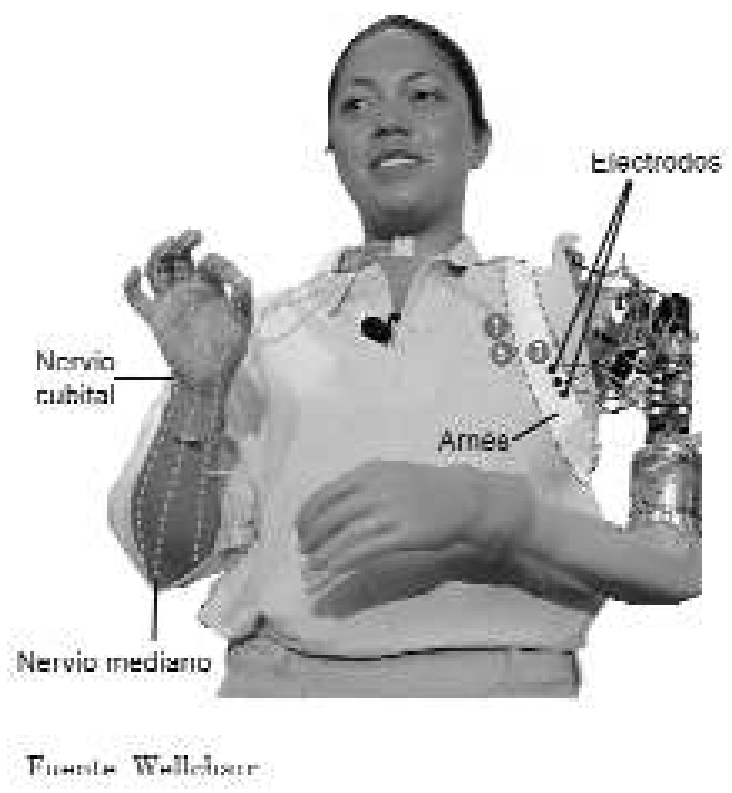

Figura 9

Implante cerebral

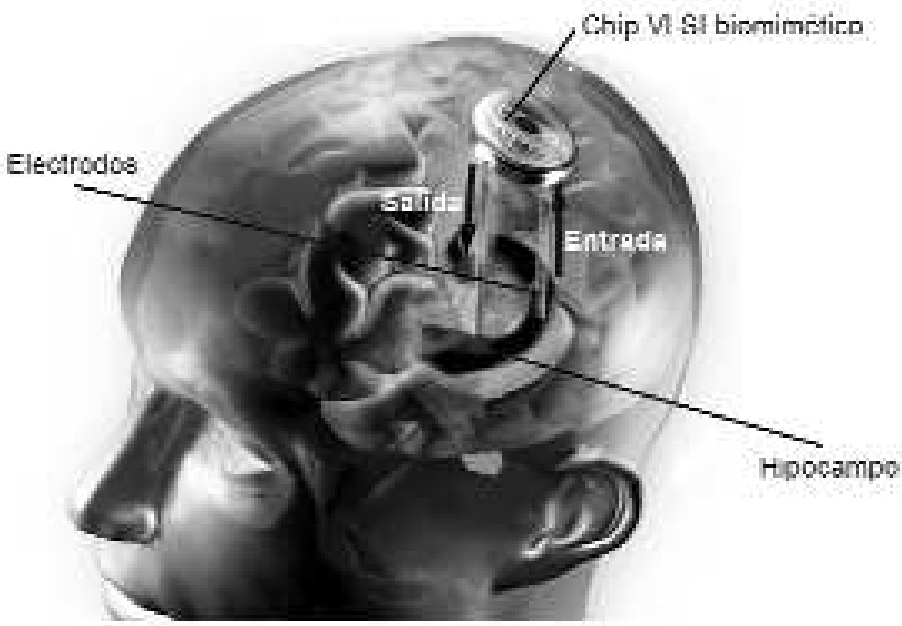

Fumme: Nruynl porfecsia. 
En la figura 10 se muestra la estructura del microchip de muy alta escala de integración VLSI (Very Large Scale Integration) en el cual se puede observar el área correspondiente al circuito de detección de impulsos, el generador de respuesta, el procesador de actualización polinómica, el circuito de temporización y las interfaces analógicas/digitales A/D y D/A para reemplazar unos 100 circuitos del hipocampo.

El Laboratorio de Ingeniería y Biomedicina de la Universidad del Sur de California ha desarrollado interfaces de dos y tres dimensiones, en las que mediante estimulación electrónica se puede conseguir actividad no espontánea y espontánea, ya sea con una o con múltiples entradas/salidas, para restaurar la memoria de pacientes con daño cerebral.

Las investigaciones incluyen el desarrollo de modelos matemáticos del sistema nervioso de mamíferos (monos y ratas), la relación de los procesos celular/molecular, funciones del sistema, conducta aprendida y la incorporación de estas funciones en el hardware del microchip biomimético. Se estima que en los próximos cinco años se realizará el primer implante en humanos.

\section{Figura 10 \\ Dispositivo VLSI biomimético}

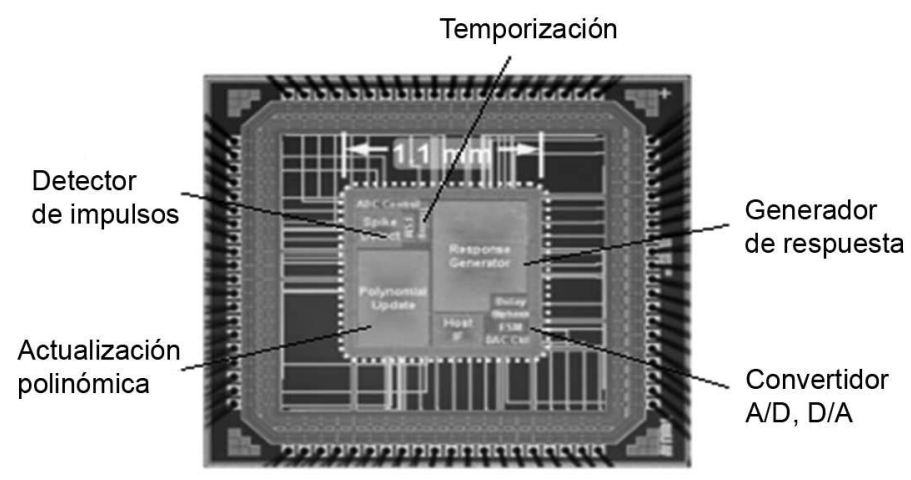

Fuente: Neural prótesis. 


\section{CONCLUSIONES}

La línea de investigación para restaurar algunos sentidos perdidos como el oído, la visión, la pérdida de memoria, e incluso reemplazar otras partes del cuerpo usando dispositivos artificiales o neuroprótesis, avanza en forma constante. Aunque los avances tecnológicos en este campo son muy amplios, quedan obstáculos por vencer. Se han creado modelos matemáticos biológicamente realistas de las funciones del cerebro, producido microchips biomiméticos que incorporan estos modelos y los integran al sistema nervioso central, desarrollado materiales que garantizan el aislamiento eléctrico extremo de los implantes, mejorado la duración y la calidad del material de los componentes, sin embargo la biocompatibilidad entre tejidos humanos y electrodos varía en función de cada persona.

\section{BIBLIOGRAFÍA}

Arbor, Ann. "New cochlear implant could improve hearing" [en línea]. University of Michigan, 6 de febrero del 2006. <http://www.umich. edu/news/index.html?Releases/ 2006/Feb06/r020606a>. [Consulta: 20 de febrero del 2008]

Berger, Theodore W. et al. "Restoring lost cognitive function" [en línea]. IEEE Engineering in Medicine and Biology Magazine. SeptiembreOctubre del 2005. <http://www. neural-prosthesis.com/doc/Restor ing $\% 20$ Lost $\% 20$ Cognitive $\% 20$ Function $\% 20$ - \% 20IEEE $\%$ 20Sep\%202005.pdf>. [Consulta: 11 de abril del 2008]

Doheny Eye Institute News. "Doheny Eye Institute Researchers to Test Next Generation of Retinal Implant" [en línea]. Doheny Eye Institute. 1 de marzo del 2007. <http://www.doheny.org/ news/retinal_ implant_news.html>. [Consulta: 20 de febrero del 2008]

Loizou, Philipos C. "Speech processing in vocoder-centric cochlear implants" [en línea]. University of Texas at Dallas. <http://www. utdallas.edu/ loizou/cimplants/chap_loizou_review2006.pdf $>$. [Consulta: 20 de febrero del 2008]

—. "Introduction to cochlear implants" [en línea]. IEEE Signal Processing Magazine, pages 101-130. Septiembre de 1998. $<$ http://www.utdallas.edu/ loizou/cimplants/tutorial/tutorial.htm> [Consulta: 20 de febrero del 2008] 
Merck Sharp \& Dohme. "Trastornos de los nervios periféricos" [en línea]. Manual Merk. <http://www.msd.es/publicaciones/mmerck_hogar/ seccion_06/seccion_06_070.html>. [Consulta: 20 de febrero del 2008]

Neural Prótesis News. "Concept of biomimetic implants for the brian" [en línea]. Neural Prótesis. <http://www.neural-prosthesis.com/index. html>. [Consulta: 20 de febrero del 2008]

Passmore R. y Robson J. S. (ed). Tratado de enseñanza integrada de la medicina. Tomo I. Barcelona: Científico-Médica, 1971.

University of Medicine and Dentistry of New Jersey. "What is a cochlear implant" [en línea]. <http://www.theuniversityhospital.com/cochlearweb/cochlearabout/whatiscochlear.htm>. [Consulta: 22 de febrero del 2008]

University of Bonn: "Intelligent retinal prosthesis at Hanover trade fair" [en línea]. <http://www1.unibonn.de/pressDB/jsp/pressemittei lungsdetails.jsp?detailjahr $=2007$ \&detail $=114>$. [Consulta: 27 de marzo del 2008]

Páginas web

Hearingloss [en línea]. <http://www.hearingloss-wa.org>.

Medgadget [en línea]. <http://medgadget.com>.

Second Sight [en línea]. <http://www.2-sight.com>.

Seingwithsound [en línea]. <http://www.seeingwithsound.com>.

Wheelchair [en línea]. <http://www.electric-wheelchair-on.net>.

Wikipedia [en línea]. <http://en.wikipedia.org>. 